\title{
CONTRACTIONS WITHOUT CYCLIC VECTORS ${ }^{1}$
}

\author{
BELLA SZ.-NAGY AND CIPRIAN FOIAS
}

\begin{abstract}
It is proved that if $T$ is a completely nonunitary contraction on Hilbert space such that $T^{* n}$ does not converge strongly to 0 as $n \rightarrow \infty$, there is an integer $N>0$ so that none of the powers $T^{* m}$ with $m \geqslant N$ has a cyclic vector. Both conditions on $T$ are essential, and the integer $N$ is not universal, i.e., it depends on $T$.
\end{abstract}

In this note we are concerned with contractions $T$ of Hilbert space $\mathcal{H}$. In previous papers [3] we considered conditions which assure the existence of cyclic vectors for $T$ (or $T^{*}$ ). Here we shall deal with a converse question, namely, to find contractions without cyclic vectors.

1. First let us recall [ 3 or $\mathbf{4}$, Chapter II] that if $U$ and $U_{+}$are the minimal unitary and isometric dilations of $T$, acting on the spaces $\mathscr{K}$ and $\mathscr{K}_{+}$, respectively $\left(\mathcal{K} \subset \mathcal{K}_{+}\right.$ $\subset \mathscr{K})$, and if $\mathscr{R}$ is the subspace of $\mathscr{K}_{+}$on which $U_{+}$is unitary, i.e., the subspace

$$
\Re=\bigcap_{n=0}^{\infty} U_{+}^{n} \mathscr{K}_{+},
$$

then $\mathscr{R}$ reduces both $U_{+}$and $U$ to the unitary operator $R=U_{+} \mid q R$. Moreover, setting $X=P_{C R} \mid \mathcal{H}$ and $\mathscr{X}=\overline{P_{G R} \mathcal{K}}$ (where $P_{C R}$ denotes the orthogonal projection of $\mathcal{K}$ onto $Q R$ ), we have

$$
X T^{*}=R^{*} X
$$

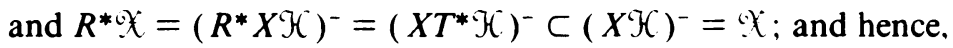

$$
\text { ox } \subset R \text { 次. }
$$

Because of the minimality of $U_{+}$, and since $Q R$ reduces $U_{+}$to $R$, we also have

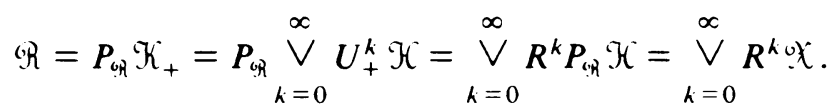

Taking (3) into account we infer that the sequence $\left\{R^{k} \cup\{\}_{k=0}^{\infty}\right.$ of subspaces of $h_{+}$ is increasing so the last member of (4) is equal to $\bigvee_{j=1}^{\infty} R^{k, 0}$ for any sequence of integers $k_{j} \rightarrow \infty($ as $j \rightarrow \infty)$; i.e.,

$$
\text { or }=\bigvee_{j=1}^{\infty} R^{k,() X .}
$$

Received by the editors May 4, 1982.

1980 Mathematics Subject Classification. Primary 47A15, 47A20.

Kel' words and phrases. Contraction. cyclic vector, unitary and isometric dilation.

'This paper was written during the stay of the first author as a guest professor at Indiana University in Bloomington, January-May. 1982. 
2. Let $\left\{E_{t}\right\}_{0 \leqslant t<2 \pi}$ be the spectral family for $R$, i.e., $R=\int_{0}^{2 \pi} e^{i t} d E_{t}$, and let $\{E(A)\}$ be the sectral measure associated with $\left\{E_{t}\right\}$, where $A$ runs over all Borel subsets of $[0,2 \pi)$; i.e., $E(A)=\int_{0}^{2 \pi} \chi_{A}(t) d E_{t}$.

Denoting by $|A|$ the Lebesgue measure of $A$, and by $\mathbf{C}$ complementation with respect to $[0,2 \pi)$, let us define

$$
\alpha_{T}=\inf \{|A|: E(\mathbf{C} A)=0\} .
$$

Lemma. (a) The infimum $\alpha_{T}$ is attained for some $A=A_{x}$ with $E\left(\mathbf{C} A_{x}\right)=0$.

(b) If the contraction $T$ is completely nonunitary and $T^{* n}$ does not tend to 0 (strongly) as $n \rightarrow \infty$, then $\alpha_{T}>0$.

Proof. Choose a minimizing sequence $\left\{A_{n}\right\}_{1}^{\infty}$ of Borel subsets of $[0,2 \pi)$, i.e. such that $E\left(\mathbf{C} A_{n}\right)=0$ and $\left|A_{n}\right| \rightarrow \alpha_{T}$. Then the set

$$
A_{\infty}=\bigcap_{1}^{\infty} A_{n}=\mathbf{C}\left(\bigcup_{1}^{\infty} \mathbf{C} A_{n}\right)
$$

obviously satisfies the requirements of (a).

If the contraction $T$ is completely nonunitary, then Theorem II.6.4 of [3] tells us that the minimal unitary dilation $U$ of $T$ has spectral measure absolutely continuous with respect to Lebesgue measure; and this property of $U$ is obviously inherited by $R=U \mid$ ? R. Thus $|A|=0$ implies $E(A)=0$. In particular, if $\alpha_{T}=\left|A_{x}\right|=0$, then $E\left(A_{x}\right)=0$. Since, on the other hand, $E\left(\mathbf{C} A_{x}\right)=0$, this leads to the conclusion $E[0,2 \pi)=0$ i.e.,,$h=\{0\}$. This in turn contradicts, by virtue of Theorem II.2.1 of [4], the assumption $T^{* n} \nrightarrow 0$. Thus part (b) of the Lemma is also proven.

3. THEOREM. Let $T$ be a completely nonunitary contraction on $:$ for which $T^{* n}$ does not tend to 0 (strongly) as $n \rightarrow \infty$. Then for all $m$ large enough, namely for $m>2 \pi / \alpha_{T}, T^{* m}$ has no cyclic vector in $:$.

Proof. Let us assume that, contrary to what was asserted, there exists a cyclic vector $h$ for $T^{* m}$. i.e., such that $\bigvee_{n=0}^{x}\left(T^{* m}\right)^{n} h=: r^{\text {. Then }}$. The have for $r=X h$, taking into account also the fact that (1) implies $X T^{* n}=R^{* n} X(n=0,1,2 \ldots)$.

$$
\bigvee_{h-0}^{\infty}\left(R^{* m}\right)^{h} r=\bigvee_{h=0}^{\infty} X\left(T^{* m}\right)^{h} h=(X ;)^{-}=k \text {. }
$$

On the other hand, applying (5) with the sequence of exponents $m j(j=0,1, \ldots)$ we have, by (7).

$$
\left(h=\bigvee_{1}^{\infty} R^{m / \cdots}=\bigvee_{1, h=0}^{\infty} R^{m(1, h)} r=\bigvee_{n=-\infty}^{\infty}\left(R^{m}\right)^{n} r .\right.
$$

Now consider any Borel set $A$ in $[0,2 \pi)$ for which $(E(A) r, r)=(r, r)$, i.e.. $E(\mathbf{C} A) r=0$. Since $R$ commutes with its spectral measure we have $E(\mathbf{C} A) q(A) r=$ $q(A) E(\mathbf{C} A) r=0$ for every trigonometric polynomial $q(R)$ of $R$. Since by virtue of (8) every $r^{\prime} \in$ ' $h$ is the (strong) limit of some sequence $\left\{q_{n}(R) r\right\}_{n}$, we have $E(\mathbf{C} A) r^{\prime}$ $=0$ for every $r^{\prime} \in\left(R ;\right.$ and thus $E(\mathbf{C} A)=0, E(A)=I_{c h}$. Therefore. $|A| \geqslant \alpha_{T}$. 
Moreover, since $s=\left(\int_{0}^{2 \pi} t d E_{t}\right) r \in \mathscr{R}$, it follows from (8) that there exists a sequence $\left\{p_{n}(z)\right\}_{n=1}^{\infty}\left(z=e^{i t}\right)$ of trigonometric polynomials such that $p_{n}\left(R^{m}\right) r \rightarrow s$. that is,

$$
\int_{0}^{2 \pi}\left|p_{n}\left(e^{i m t}\right)-t\right|^{2} d\left(E_{t} r, r\right)=\left\|p_{n}\left(R^{m}\right) r-s\right\|^{2} \rightarrow 0, \text { as } n \rightarrow \infty .
$$

Replacing the sequence $\left\{p_{n}\right\}$, if necessary, by an appropriate subsequence (still denoted by $\left.\left\{p_{n}\right\}\right)$ we have even pointwise convergence

$$
p_{n}\left(e^{i m t}\right) \rightarrow t, \quad \text { as } n \rightarrow \infty,
$$

almost everywhere with respect to the measure $(E(\cdot) r, r)$, i.e., on a (necessarily Borel) set $A_{m}$ of full $(E(\cdot) r, r)$-measure: $\left(E\left(A_{m}\right) r, r\right)=(r, r)$. As it was shown above this implies

$$
\left|A_{m}\right| \geqslant \alpha_{T} \text {. }
$$

As the functions $p_{n}\left(e^{i m t}\right)$ are periodic in $t$, with period $2 \pi / m$, relation (9) implies that $A_{m}$ has the property

(11) if $t_{1}, t_{2} \in A_{m}$ are such that $t_{1}-t_{2}=2 \pi k / m$ for some integer $k$, then $t_{1}=t_{2}$.

Consider the sets

$$
B_{k}=A_{m} \cap\left[\frac{2 \pi}{m} k, \frac{2 \pi}{m}(k+1)\right) \quad(k=0,1,2 \ldots m-1)
$$

and their copies $B_{k}^{\prime}=B_{k}-2 \pi k / m$ shifted into $[0,2 \pi / m)$. From (11) we get that the sets $B_{k}^{\prime}$ are mutually disjoint. Hence,

$$
\frac{2 \pi}{m} \geqslant\left|\sum_{k=0}^{m-1} B_{k}^{\prime}\right|=\sum_{k=0}^{m-1}\left|B_{k}^{\prime}\right|=\sum_{k}^{m}\left|B_{k}\right|=\left|A_{m}\right| .
$$

In view of (10) this contradicts the choice of $m$ in the Theorem.

This completes the proof.

4. Remarks. (a) The Theorem does not hold if the assumption that $T$ be completely nonunitary is dropped.

Indeed, consider the operator $T$ on 2 -space $\mathfrak{H}_{2}$ given by the matrix diag $[1, \varepsilon]$. $\varepsilon=e^{2 \pi i a}$, where $a$ is a real, irrational number. $T$ is unitary so $T^{* n}$ does not converge (strongly) to 0 as $n \rightarrow \infty$. But $T^{* m}$ has, no matter how large $m$ is, a cyclic vector. Moreover, every vector $h=\left[\begin{array}{l}a \\ h\end{array}\right]$ with $a, b \neq 0$ is cyclic for $T^{* m}(m \geqslant 1)$ : indeed $h$ and $T^{* m} h$ already span $: \mathcal{H}$ : for every $k=\left[\begin{array}{l}u \\ v\end{array}\right] \in$ ? $h$ we have $c_{0} h+c_{1} T^{* m} h=k$ for some numbers $c_{0}, c_{1}$; this is a consequence of the fact that $\varepsilon^{-m} \neq 1$.

(b) On the other hand, the mere condition that $T$ be completely nonunitary does not suffice. Indeed, the simple unilateral shift $S$ on $l^{2}$ is clearly completely nonunitary, but, for every fixed $m>2, S^{* m}$, being unitarily equivalent to $S^{*} \oplus S^{*}$ $\oplus \cdots \oplus S^{*}$ ( $m$ terms), has a cyclic vector. (See [2, Problem 126].)

(c) The Theorem cannot be improved so that a given power, say $T^{* N}$, is not cyclic for all $T$ considered. 
To prove this, consider the function

$$
\begin{gathered}
\theta(z)=\exp \left(\frac{1}{2 \pi} \int_{0}^{2 \pi} \frac{e^{i t}+z}{e^{t}-z} \log k_{N}(t) d t\right) \quad(|z|<1), \\
k_{N}(t)= \begin{cases}1 & \text { on }(2 \pi / N, 2 \pi), \\
1 / 2 & \text { on }[0,2 \pi / N] .\end{cases}
\end{gathered}
$$

This function belongs to the Hardy class $H^{\infty}$, and the radial limit $\theta\left(e^{\prime \prime}\right)=$ $\lim _{r \rightarrow 1} \theta\left(r e^{t t}\right)$ exists and satisfies $\left|\theta\left(e^{t t}\right)\right|=k_{N}(t)$ almost everywhere on $0 \leqslant t \leqslant 2 \pi$. with respect to Lebesgue measure. Hence,

$$
1-\left|\theta\left(e^{\prime t}\right)\right|^{2}=1-\left|k_{N}(t)\right|^{2}= \begin{cases}0 & \text { on }(2 \pi / N, 2 \pi), \text { a.e. } \\ 3 / 4 & \text { on }[0.2 \pi / N], \text { a.e. }\end{cases}
$$

In particular, $\theta(z)$ is a purely contractive analytic function $\left(\theta(0)=2^{-N / 2}\right)$ : thus it coincides with the characteristic function of a contraction $T$ [4, Chapter VI], which is unitarily equivalent to the operator $V_{N}$ of multiplication by $e^{\prime \prime}$ on the space $L^{2}(0,2 \pi / N)$ (Theorem V1.6.1 in [4]). Therefore, $T^{* N}$ is unitarily equivalent to the operator of multiplication by $e^{-i t}$, and so also to the operator of multiplication by $e^{\prime \prime}$. on $L^{2}(0,2 \pi)$. But this operator is cyclic (with the cyclic vector $e^{-1 / t} \in L^{2}(0.2 \pi)$. say). Thus $T^{* N}$ also has a cyclic vector.

(d) Our Theorem is an analogue of a deep result of Berger [1] concerning hyponormal operators, and was partly inspired by some comments on this result made by R. G. Douglas and C. Pearcy.

\section{REFERENCES}

1. C. Berger. Intertwined operators and the Pincus principal function. Integral Equations Operator Theory $4(1981), 1-9$.

2. P. R. Halmos, A Hilhert space problem hooh. Van Nostrand. Princeton. N. J.. 1967

3. B. Sz.-Nagy and C. Foias. Vecteurs cicliques et commutaticite des commutants. I. II. Acta Sci. Math. 32 (1971). 177-183: 39 (1977), 169-174.

4. B. Sz.-Nagy and C. Foias. Harmonic analysis of operators on Hilhert space. North-Holland, Amsterdam and Akademiai Kiadó. Budapest, 1970.

Bolyai Institute. University of Szeged. Szeged, Hungary 6720

DEPARTMENT OF MATHEMATICS. INDIANA UNIVERSITY. BLOOMINGTON. INDIANA 47401 